> Les maladies inflammatoires chroniques, polyarthrite rhumatoïde, spondylarthrite ankylosante, lupus érythémateux systémique, psoriasis, maladie de Crohn, ou sclérose en plaques, ont en commun l'implication de cytokines proinflammatoires dans les manifestations systémiques et articulaires. Ces molécules constituent autant de cibles pour le développement de molécules antagonistes thérapeutiques, dont les anticorps monoclonaux (Acm). La polyarthrite rhumatoïde est à ce titre emblématique puisqu'elle représente le premier exemple de maladie non cancéreuse dans laquelle un Acm thérapeutique dirigé contre le TNF $\alpha$ (tumor necrosis factor alpha) a été utilisé. Depuis, d'autres Acm ont été proposés, ciblant d'autres cytokines, ou depuis récemment, les cellules effectrices immunes et leurs voies de communication, dont les lymphocytes T. Si un bénéfice à long terme de tels traitements est souvent acquis, il doit être confronté à l'évaluation d'autres paramètres comme le risque infectieux, la hiérarchie d'utilisation par rapport aux autres thérapeutiques disponibles, ou encore leur coût. <

\section{Les anticorps monoclonaux dans les maladies immunes inflammatoires chroniques}

Luca Semerano, Marie-Christophe Boissier

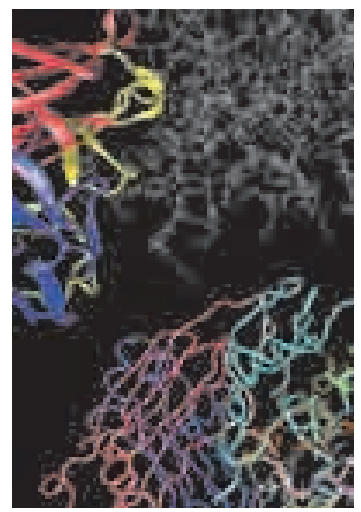

Université Paris 13, EA4222, Li2P, Bobigny, France. Assistance publique-hôpitaux de Paris, Service de rhumatologie, hôpital Avicenne, 125 , rue de Stalingrad, 93017 Bobigny Cedex, France. marie-christophe.boissier@ avc.aphp.fr

exemple de maladie non cancéreuse dans laquelle un Acm thérapeutique dirigé contre le TNF $\alpha$ (tumor necrosis factor alpha) a été utilisé à large échelle; c'est certainement dans cette indication également que le plus grand nombre de cibles a été identifié pour ensuite aboutir à un développement thérapeutique très significatif. Nous évoquerons les principales stratégies utilisant les Acm actuellement développées dans les maladies inflammatoires chroniques. Le clinicien dispose parfois aussi en parallèle, dans ces mêmes indications, d'autres biothérapies ciblées n'utilisant pas les $A c m$ (comme l'étanercept, l'anakinra ou

$\rightarrow$ voir J. Sibilia, L'étiologie des maladies inflammatoires chroniques d'origine auto-immune comme la polyarthrite rhumatoïde (PR), la spondylarthrite ankylosante (SPA), le lupus érythémateux systémique (LES), le psoriasis, la maladie de Crohn, ou la sclérose en plaques (SEP) reste énigmatique. Les antigènes responsables de ces maladies sont mal définis comme l'est la hiérarchie de leur implication. $\varepsilon n$ revanche, notre connaissance détaillée des mécanismes intermédiaires d'entretien de l'inflammation et de la destruction tissulaire a permis d'identifier des cibles puis des molécules thérapeutiques, et notamment des anticorps monoclonaux (Acm) (Tableau I). L'exemple de la PR est emblématique du développement de ces stratégies: c'est une maladie fréquente, responsable d'une inflammation polyarticulaire avec destruction, entraînant douleur, handicap et mortalité accrue. La PR a été le premier

\section{Anticorps anti-TNF}

Les gènes codant le TNF $\alpha$ et l'interleukine-1 (IL-1) ont été séquencés en 1984 [1] et les cytokines correspondantes identifiées comme les médiateurs de l'inflammation et de la destruction articulaire [2, 3]. La responsabilité du TNF $\alpha$ et de l'IL-1 a été ensuite clairement démontrée dans les modèles expérimentaux de maladies articulaires et dans la PR. Les souris transgéniques

${ }^{1}$ Etanercept ( $\left(\varepsilon_{n} d r e l^{\circledR}\right)$ est un récepteur du TNF soluble ; abatacept est une protéine de fusion qui interrompt le signal de costimulation lymphocytaire $T$ induit via la voie $\mathrm{CD} 28-\mathrm{CD} 80 / \mathrm{CD} 86$. 


\begin{tabular}{|c|c|c|c|}
\hline Cible & Nom & Développement & $\begin{array}{c}\text { Indic. } \\
\text { principales } \\
\text { (dans ou hors AMM) }\end{array}$ \\
\hline TNF $\alpha$ & Infliximab & AMM & $\begin{array}{l}\text { PR, SPA, psoriasis, } \\
\text { arthrite juvénile }\end{array}$ \\
\hline TNF $\alpha$ & Adalimumab & AMM & $\begin{array}{l}\text { PR, SPA, psoriasis, } \\
\text { arthrite juvénile }\end{array}$ \\
\hline TNF $\alpha$ & Golimumab & AMM & $P R$ \\
\hline TNF $\alpha$ & Certolizumab-pégol & AMM & $P R$ \\
\hline IL-1 $\beta$ & AMG 108 & Phase II & $P R$ \\
\hline IL-5 & Mépolizumab & Phase III & Synd. hyperéosinophile \\
\hline IL-6R $\alpha$ & Tocilizimumab & AMM & $\begin{array}{l}\text { PR, maladie de Castleman, } \\
\text { maladie de Still }\end{array}$ \\
\hline IL-15 & HuMax IL-15 & Phase II & PR \\
\hline IL-18 & Anti-IL-18 Mab & Phase I & PR \\
\hline IL-23/IL-12 & Ustékinumab & AMM & Psoriasis, maladie de Crohn \\
\hline RANK-L & Dénosumab & Phase III & PR (destruction osseuse) \\
\hline BLyS & Bélimumab & Phase II & PR, LES \\
\hline CD52 & Alemtuzumab & Phase II & SEP \\
\hline CD20 & Rituximab & AMM & $P R$, LES, auto-immunité $B$ \\
\hline CD20 & Ocrélizumab & Phase III & PR, LES \\
\hline CD20 & Ofatumumab & Phase III & $P R$ \\
\hline CD22 & Épratuzumab & Phase II & LદS \\
\hline $\lg \varepsilon$ & Omalizumab & AMM & Asthme, allergie \\
\hline$\alpha 4$-intégrine & Natalizumab & AMM & SEP \\
\hline
\end{tabular}

Tableau I. Anticorps monoclonaux dans les maladies inflammatoires. AMM: autorisation de mise sur le marché ; LES : lupus érythémateux systémique ; SPA : spondylarthrite ankylosante; PR : polyarthrite rhumatoïde; SEP: sclérose en plaques. RANK-L (ou ostéoprotégérine) : receptor activator of nuclear factor kappa $B$.

surexprimant le TNF $\alpha$ sont atteintes d'une inflammation et d'une destruction polyarticulaires. Ces découvertes ont permis de définir ces deux cytokines comme des cibles thérapeutiques intéressantes et les essais cliniques des molécules interférant avec leur action ont confirmé de façon éclatante cette hypothèse. L'infliximab, premier Acm anti-TNF $\alpha$ utilisé dans les années 1990, démontrait son efficacité dans la PR [4], en monothérapie mais surtout en association avec un traitement classique comme le méthotrexate. L'adalimumab, Acm anti-TNF $\alpha$ entièrement humanisé, induisait les mêmes résultats positifs quelques années plus tard. De façon concomitante, l'étanercept, forme soluble du récepteur du TNF $\alpha$ couplée à un fragment $F c$, faisait aussi preuve d'une efficacité franche dans la PR (Figure I).
Les effets thérapeutiques bénéfiques de ces antagonistes du TNF $\alpha$ ont été observés ensuite dans les spondylarthrites, leur efficacité s'exerçant à la fois sur les arthrites périphériques et les atteintes rachidiennes. Outre l'avancée thérapeutique que constituait la mise à disposition, en pathologie humaine, de ces anti-TNF $\alpha$, ils ont aussi eu pour conséquence de conforter l'hypothèse d'un rôle clé du TNF dans la pathogénie de certaines maladies inflammatoires, comme la spondylarthrite ankylosante (SPA), rôle suspecté mais non démontré comme étant déterminant. On pouvait en effet penser que la redondance du réseau des cytokines ne permettrait pas de bloquer l'inflammation en ciblant une seule cytokine.

L'effet thérapeutique sur l'inflammation était franc et souvent rapide (2 à 6 semaines en moyenne après l'institution du traitement), et associé à un ralentissement notable de la destruction articulaire (évalué dans les études prospectives à long terme) ; le rapport bénéfice/risque était finalement très favorable malgré le rôle majeur du TNF $\alpha$ dans la défense anti-infectieuse et antitumorale qui pouvait faire craindre des effets délétères de type infectieux ou tumoral $[5](\rightarrow)$. II faut $(\rightarrow)$ voir R. Rigal et
cependant insister sur le fait que le risque de lymphome doit faire l'objet d'études à long et très long terme. Quant au risque infectieux, il est réel, particulièrement celui de développer une tuberculose mais aussi d'autres infections parfois sévères, et ce d'autant que les patients sont souvent immunodéprimés par leur maladie et un traitement corticoïde associé. Une sélection rigoureuse des malades rend très rare l'exacerbation d'une tuberculose latente chez les patients traités par anti-TNFa. Cette éventualité reste cependant une complication redoutée du thérapeute. Pour chaque patient le risque de tuberculose est évalué : recherche d'antécédents de tuberculose, examen clinique, radiographie du thorax, intra dermo-réaction à la tuberculine; au moindre doute un scanner thoracique et la recherche de bacilles de Koch par tubages gastriques sont réalisés; au moindre risque, un traitement antituberculeux est institué avant tout traitement par des antagonistes du TNF. 


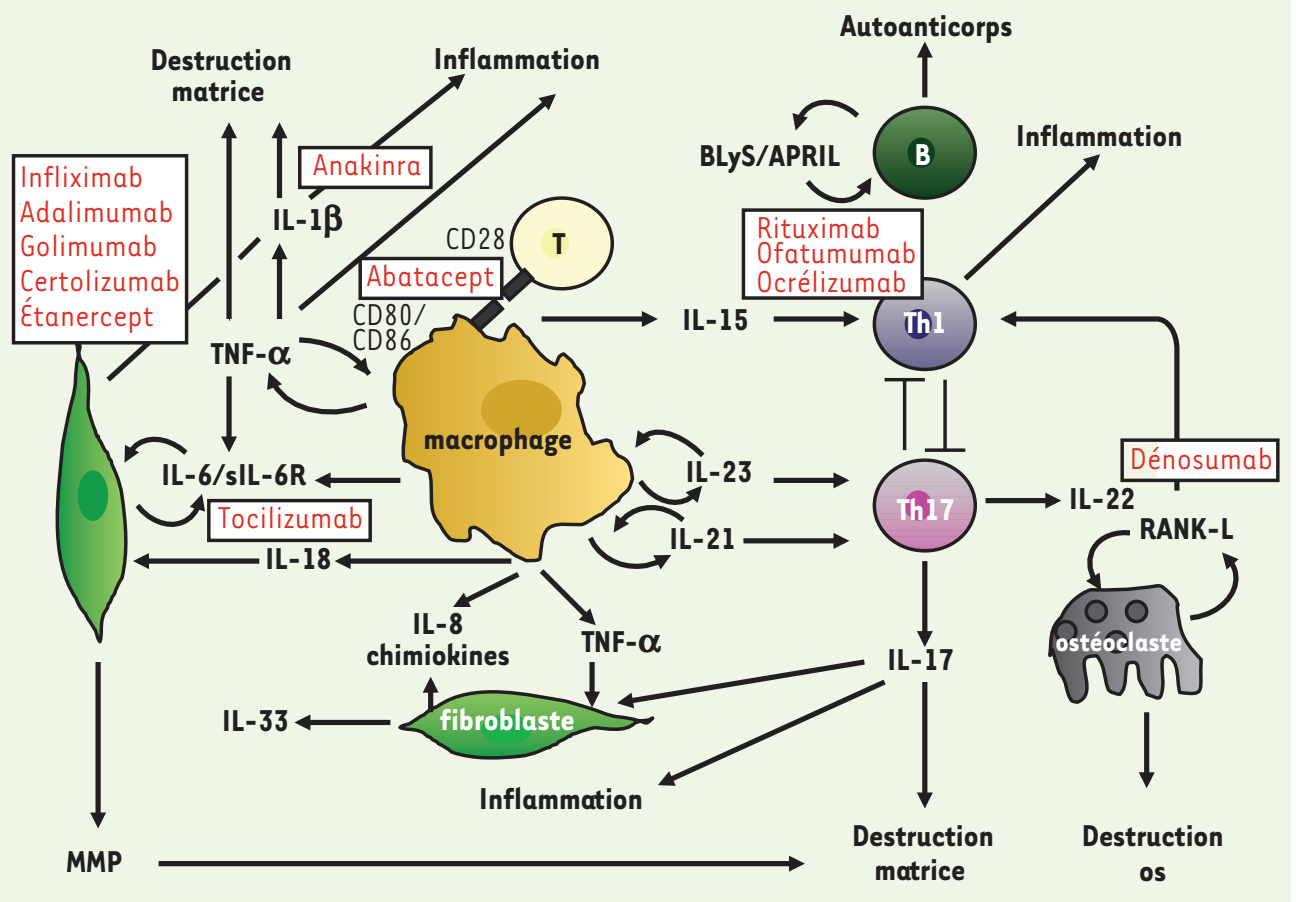

Figure 1. Voies pathogéniques de la polyarthrite rhumatoïde avec les principaux acteurs. Les cytokines majeures et les cellules cibles concernées font l'objet de développements thérapeutiques spécifiques. Les principaux traitements ciblés disponibles ou en cours de disponibilité sont représentés en rouge à proximité de leur cible: infliximab, adalimumab, golimumab, certolizumab sont des Acm anti-TNF $\alpha$; le tocilizumab est un Acm anti-IL-6R; le rituxumab est un Acm antiCD20, le dénosumab est un Acm anti-RANK-L. L'anakinra, l'étanercept et l'abatacept, qui ne sont pas des Acm, sont respectivement des antagonistes spécifiques de l'IL-1, du TNF $\alpha$ et de CD80/CD86. MMP : métalloprotéinases matricielles.

On considère qu'aujourd'hui plus d'un million de patients ont bénéficié d'un traitement anti-TNF $\alpha$ dans la PR, la SPA, mais aussi la maladie de Crohn (où seuls les Acm anti-TNF $\alpha$ sont efficaces mais pas l'étanercept) [6]. Le bénéfice à long terme de tels traitements est acquis, mais la rémission complète (dont la définition est encore sujet de controverse) est inconstante et justifie le développement d'autres stratégies de blocage de l'inflammation.

\section{Anticorps dirigés contre d'autres cytokines}

Le blocage de l'IL-1 était une stratégie théoriquement aussi logique que celle du blocage du TNF $\alpha$. Dans l'inflammation chronique articulaire, les modèles expérimentaux tendent à montrer que l'IL-l agit plus en aval que le TNF $\alpha$ et favorise directement et de façon plus importante la destruction ostéocartilagineuse. Le développement clinique d'un traitement ciblant I'IL-1 a abouti à la conception non pas d'un Acm mais d'un antagoniste du récepteur à l'IL-l (anakinra) qui, à l'usage, a révélé un effet clinique moins spectaculaire que celui des anti-TNF $\alpha$ sur l'inflammation et la destruction articulaires. Depuis peu, un Acm anti-IL-1 $\beta$ fait l'objet d'essais cliniques, cette fois plus prometteurs. II faut noter que le dénosumab, Acm dirigé contre RANK- $L^{2}$ (qui est

2 «RANKL est une protéine membranaire synthétisée par les ostéoblastes et les lymphocytes T activés, qui se lie à son récepteur RANK présent à la surface des précurseurs ostéoclastiques. Ce récepteur est également exprimé par les monocytes, les cellules dendritiques, les fibroblastes et les lymphocytes B et T. L'interaction de RANKL avec son récepteur RANK est indispensable à l'ostéoclastogenèse, mais également à la survie et à l'activation des DC. Dans ce dernier cas, c'est le contact entre les lymphocytes T, qui synthétisent RANKL, et les DC exprimant le récepteur RANK, qui permet son action. » (reproduit de [14]) responsable de l'activation ostéoclastique dans l'ostéoporose et les maladies inflammatoires comme la PR) est aussi en phase d'essais cliniques [7].

L'IL-6, induite par le TNF $\alpha$ et I'IL-1, connue pour son rôle d'activation des protéines hépatiques de la phase aiguë de l'inflammation, est la cytokine inflammatoire la plus abondante dans l'articulation. Le tocilizumab, Acm dirigé contre la chaîne $\alpha$ du récepteur à l'IL-6, est d'une efficacité comparable à celle des Ac anti-TNF $\alpha$ dans la PR.

Des développements nombreux sont en cours d'exploration (Tableau I), comme les Acm anti-interféron- $\alpha$ ou anti-IL-10 dans le LES.

\section{Anticorps ciblant des lymphocytes}

Les lymphocytes T jouent un rôle essentiel dans l'apparition et le maintien des manifestations cliniques des maladies immunes inflammatoires [8]. Les résultats initiaux très prometteurs des Acm anti-CD4 dans la PR n'ont pas été confirmés dans les études contrôlées. Des essais avec un Acm anti-CD $52^{3}$ (alemtuzumab) n'ont pas permis de conclure, chez l'homme, à une

${ }^{3} \mathrm{CD} 52$ : antigène membranaire, fixé via un groupement glycosylphosphatidylinositol et exprimé par tous les lymphocytes et par les cellules épithéliales du tractus génital mâle. 
efficacité franche. Toutefois des résultats prometteurs sont notés dans la sclérose en plaques (SEP). Le blocage des voies de costimulation des lymphocytes $T$ a conduit au développement, non pas d'un Acm, mais de l'abatacept: il s'agit d'une protéine de fusion soluble couplant le domaine extracellulaire du CTLA4 (Cytotoxic T-lymphocyte antigen 4, aussi défini comme CD152) humain à un fragment de Fc $\gamma 1$. Elle joue un rôle inhibiteur en se liant aux molécules CD80/ CD86, inhibant leur interaction avec CD28, et interférant de ce fait avec le second signal d'activation des lymphocytes. Cette molécule est utilisée dans la PR.

Les Acm dirigés contre les lymphocytes B sont également efficaces dans plusieurs maladies inflammatoires comme la PR et le LES. Le rituximab, un Acm antiCD20 développé pour le traitement des lymphomes à cellules $B(\rightarrow)$, est

$\rightarrow)$ voir G. Cartron et J.F. Rossi, page 1085 utilisé avec succès dans la PR en association avec le méthotrexate. Une série de deux administrations par perfusions espacées de 15 jours peut être efficace pendant une durée prolongée parfois supérieure à un an. Le rituximab est également efficace dans les cryoglobulinémies, le purpura thrombopénique idiopathique, les anémies hémolytiques auto-immunes, les vascularites associées aux ANCA (anti-neutrophil cytoplasmic antibodies), les pemphigus sévères. Des molécules dirigées contre les molécules d'activation des lymphocytes B (anti-BLyS) sont également testées dans la PR et le LES.

\section{Hiérarchie thérapeutique, questions non résolues}

Le développement des thérapeutiques ciblées est le résultat direct de la recherche physiopathologique réalisée en amont. II apparaît clairement que la découverte de nouvelles cibles potentielles et les progrès de l'immunopharmacologie laissent prévoir d'importants développements thérapeutiques dans les maladies inflammatoires chroniques [9]. Cependant, si l'on ne considère que l'exemple de la PR, le préalable à la mise sur le marché par l'industrie pharmaceutique de nouvelles molécules est la démonstration d'une efficacité au moins égale à celle des médicaments déjà disponibles, un prérequis méthodologiquement complexe dans cette maladie: à court terme, les traitements ciblés disponibles ont une efficacité satisfaisante chez 60 à $70 \%$ des patients réfractaires à au moins un traitement classique.

D'autres paramètres entrent en ligne de compte et notamment des paramètres économiques. Le coût annuel des traitements par Acm dans les indications décrites précédemment est pour la plupart d'entre eux de l'ordre de $15 \mathrm{~K}$ euros par patient, une administration pendant plusieurs années étant à prévoir [10]. C'est aussi dans ce contexte que se développent les alternatives aux traitements par Acm : conception d'inhibiteurs naturels comme les récepteurs solubles de cytokines pro-inflammatoires ou d'inhibiteurs de molécules de stimulation, ou encore vaccins anti-cytokines.

\section{Paramètres de réponse au traitement}

Les Acm sont d'un apport considérable pour les patients concernés. Certes leur effet clinique, dont l'appréciation dépend étroitement de la définition des critères d'efficacité, est inconstant. Les échecs sont de plusieurs natures: un échec immédiat (résistance primaire) identifie un patient non répondeur; cette situation peut refléter l'incomplète compréhension des affections en cause. Par exemple certaines PR sont très sensibles aux anti-TNF, d'autres totalement résistantes, indépendamment de leur sévérité clinique. Un échec survenant après plusieurs mois ou années d'efficacité (résistance secondaire) peut indiquer l'apparition d'anticorps anti-médicaments (anti-anti-TNF) dont la détection s'affine progressivement $(\rightarrow)$ voir L. Manache $(\rightarrow)$. Dans d'autres cas, la résistance et al., page 1063 secondaire peut signifier une adaptation du réseau cellulaire et cytokinique. L'intérêt du dosage des Acm est en cours d'évaluation [11]. La prédiction de l'efficacité thérapeutique d'un Acm n'est guère possible en pratique dans les maladies inflammatoires chroniques en l'absence de consensus. Les études de pharmacogénomique sont nombreuses: l'étude du polymorphisme du promoteur du TNF $\alpha$ était prometteuse mais son intérêt ne fait pas l'unamimité [12]. L'évaluation quantitative de la cible à atteindre est également peu corrélée à l'efficacité observée, d'où le faible intérêt du seul dosage sérique du TNF $\alpha$ avant l'administration d'infliximab ou le simple comptage des lymphocytes B exprimant le CD20 avant celle de rituximab. L'intérêt de la collection d'un ensemble de paramètres grâce à des dosages multiples simultanés est en cours d'étude.

\section{Choix thérapeutiques: quels arguments?}

La hiérarchie d'administration des différents traitements par Acm n'est pas établie, mais il est clair que ces traitements ont d'abord été utilisés dans les formes les plus graves des maladies inflammatoires chroniques. L'évolution rapide des pratiques fait que I'utilisation des Acm est maintenant proposée pour traiter des formes précoces de maladies (et elle est souvent associée à un traitement classique), après identification de facteurs de mauvais pronostic. C'est le cas par exemple de PR récentes dans lesquelles existe déjà une destruction articulaire, même minime. On peut prévoir que, dans un proche avenir, la simple estimation d'un risque de sévérité entraînera à proposer ces traitements dans des formes précoces, avant l'apparition de destructions tissulaires irréversibles. Le passage d'un traitement ciblé à l'autre est possible (par exemple: prescription d'un anti-TNF $\alpha$, puis de rituximab, puis de tocilizumab), et n'obéit actuellement à aucune règle étayée par des arguments cliniques. L'association de ces traitements, proposition logique, est dangereuse potentiellement: la mortalité par infection par exemple est un risque avéré majeur de l'association des anti-TNF $\alpha$ et des 
inhibiteurs de l'IL-1, ce qui contraste avec l'excellente tolérance de chacune des deux molécules utilisées séparément. La surveillance clinique à moyen et long terme des effets secondaires est une nécessité, la multiplicité des conséquences du blocage d'une voie du système immunitaire étant par essence imprévisible. Certaines pathologies exceptionnelles, comme la leucoencéphalite multifocale progressive, peuvent survenir au cours de l'évolution de désordres immunitaires chroniques et pourraient être observées lors de traitements ciblés $(\rightarrow)$. L'exemple de l'efalizumab (Acm anti-CDIla développé dans le $(\rightarrow$ ) voir $\boldsymbol{\varepsilon}$. Rigal et psoriasis) conduit à la prudence, tant dans l'inter- al., page 1135 prétation des données que dans la prescription d'un Acm dans une maladie inflammatoire. Si l'on en attend un gain, celui-ci n'est jamais certain dans ces affections complexes et graves. La recherche thérapeutique se poursuit, identifiant de nouvelles cibles, affinant les moyens de les atteindre par d'autres Acm, voire en proposant des stratégies alternatives d'immunothérapie active $[13,15]$. $\diamond$

\section{SUMMARY}

Monoclonal antibodies

in chronic autoimmune inflammatory diseases

Among chronic inflammatory diseases, rheumatoid arthritis is a common inflammatory and destructive arthropathy, characterized by the release of potent proinflammatory cytokines mostly TNF $\alpha$ and IL-1, which both mediate systemic effects and contribute to joint destruction. Many therapeutic agents have been proposed to antagonise these cytokines, among which monoclonal antibodies. Thus twenty years ago the antiTNF $\alpha$ infliximab was the first monoclonal antibody to be proposed in a non-cancerous indication, rheumatoid arthritis. Since then, several other monoclonal antibodies and/or antagonists either targeting cytokines (IL-1, IL-6, RANKL), but also immune cellular effectors $T$ and $B$ cells, have been evaluated not only in rheumatoid arthritis, but also in systemic lupus, Crohn's disease, multiple sclerosis, or ankylosing spondylitis. Clinical benefit has been unambiguously demonstrated, but before these novel molecules enter routine clinical practice, several parameters will have to be accurately documented such as their safety, long term efficacy, and economical cost. $\diamond$

\section{CONFLIT D'INTÉR̂ิTS}

Les auteurs déclarent n'avoir aucun conflit d'intérêts concernant les données publiées dans cet article.

\section{RÉFÉRENCES}

1. Pennica D, Nedwin GE, Hayflick JS, et al. Human tumour necrosis factor: precursor structure, expression and homology to lymphotoxin. Nature $1984 ; 312: 724-9$.

2. Saklatvala J, Sarsfield SJ, Townsend Y. Pig interleukin 1. Purification of two immunoogically different leukocyte proteins that cause cartilage resorption, lymphocyte activation, and fever. J Exp Med 1985 ; 162: 1208-22.

3. Henderson B, Pettifer $\varepsilon$ R. Arthritogenic actions of recombinant IL-1 and tumour necrosis factor alpha in the rabbit: evidence for synergistic interactions between cytokines in vivo. Clin Exp Immunol 1989; $75: 306-10$.

4. Elliot MJ, Maini RN, Feldmann M, et al. Treatment of rheumatoid arthritis with chimeric monoclonal antibodies to tumor necrosis factor alpha. Arthritis Rheum 1993; 36 : 1681-90.

5. Vital $\varepsilon M$, , Emery P. The development of targeted therapies in rheumatoid arthritis. J Autoimmunity $2008 ; 31: 219-27$.

6. Feldmann M, Maini RN. Role of cytokines in rheumatoid arthritis: an education in pathophysiology and therapeutics. Immunol Rev 2008; $223: 7-19$.

7. Brennan F, Mc Innes IB. Evidence that cytokines play a role in rheumatoid arthritis. J Clin Invest 2008 ; 118 : 3537-45.

8. Boissier MC, Assier $\varepsilon$, Falgarone G, et al. Shifting the imbalance from Thl/ Th2 to Th17/treg: the changing rheumatoid arthritis paradigm. Joint Bone Spine $2008 ; 75: 373-5$.

9. Scheinecker C, Redlich K, Smolen J. Cytokines as therapeutic targets: advances and limitations. Immunity $2008 ; 28: 440-4$.

10. Launois R, Payet $S$, Saidenberg-Kermanac'h N, et al. Budget impact model of rituximab after failure of one or more TNFalpha inhibitor therapies in the treatment of rheumatoid arthritis. Joint Bone Spine $2008 ; 75: 688-95$.

11. Cassinotti A, Travis S. Incidence and clinical significance of immunogenicity to infliximab in Crohn's disease: a critical systematic review. Inflamm Bowel Dis 2009 (sous presse).

12. Ranganathan $P$. An update on pharmacogenomics in rheumatoid arthritis with a focus on TNF-blocking agents. Curr Opin Mol Ther $2008 ; 10: 562-7$.

13. Delavallée L, Le Buanec $H$, Bessis N, et al. Early and long-lasting protection from arthritis in tumour necrosis factor alpha (TNFalpha) transgenic mice vaccinated against TNFalpha. Ann Rheum Dis 2008 ; $67: 1332-8$.

14. Gallois A, Mazzorana M, Vacher J, Jurdic P. Ostéo-immunologie : une vision globale et intégrée du tissu squelettique et du sytème immunitaire. Med Sci (Paris) $2009 ; 25: 259-65$.

15. Bensussan A, Bizzini B, Pouletty $P$, et al. Les kinoïdes : Une nouvelle génération de vaccins thérapeutiques. Med Sci (Paris) $2008 ; 24: 306-13$.

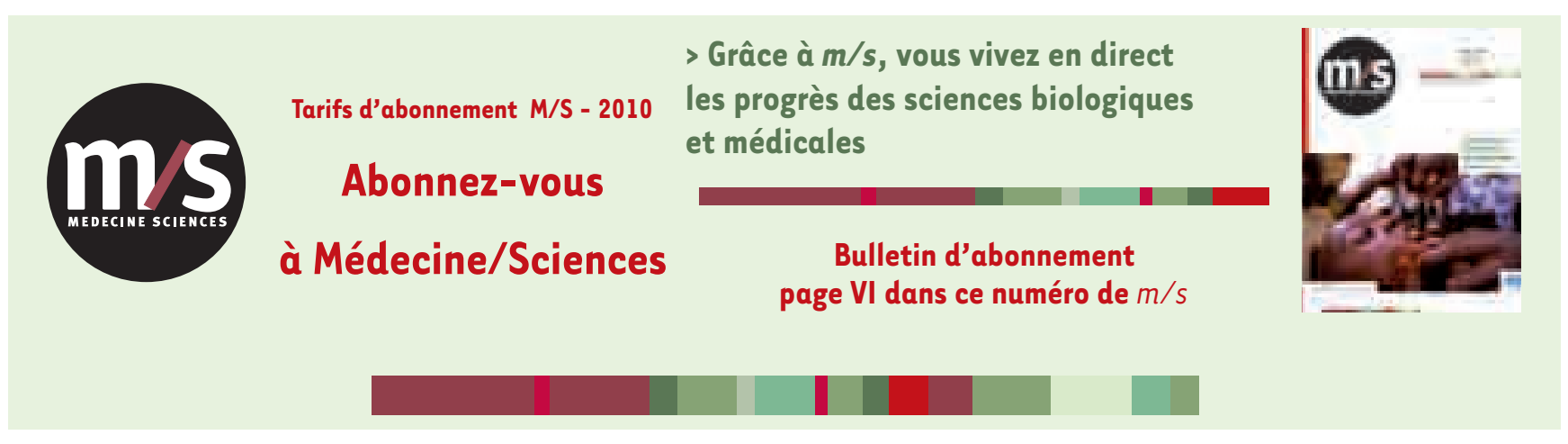

TIRÉS À PART

M.C. Boissier 